\title{
Desprendimiento seroso de retina agudo inducido por cirugía en posición prono
}

\author{
Acute serous detachment of retina after surgery in prone \\ position
}

María Carolina Sardi-Correa', Susana Cardona-Bernal ${ }^{2}$, Juan José Ortiz-Vásquez ${ }^{3}$

Fecha correspondencia:

Recibido: febrero 21 de 2020.

Revisado: mayo 26 de 2020.

Aceptado: octubre 20 de 2020.

Forma de citar:

Sardi-Correa MC, Cardona-

Bernal S, Ortiz-Vásquez JJ.

Desprendimiento seroso de retina

agudo inducido por cirugía en

posición prono. Rev CES Med.

2020; 34(3): 215-220.

Open access

(C) Derecho de autor

Licencia creative commons

Ética de publicaciones

Revisión por pares

Gestión por Open Journal System

DOl: http://dx.doi.org/10.21615/

cesmedicina.34.3.5

ISSN 0120-8705

e-ISSN 2215-9177

Sobre los autores:

1. Oftalmóloga retinóloga, Clínica Oftalmológica de Antioquia,

Medellín, Colombia.

Comparte

F日G.

\section{Resumen}

La posición en decúbito prono es utilizada en cirugía para acceder a diferentes estructuras anatómicas del paciente. Esta posición puede asociarse a pérdida visual postoperatoria en el 0,05 a $1 \%$ de las complicaciones reportadas. El desprendimiento seroso de retina es la separación de la retina neurosensorial del epitelio pigmentario de la retina por acumulación de líquido, sin daño estructural, como resultado de cambios en la permeabilidad y perfusión de la coroides. Se presenta el caso de una mujer con indicación de extracción de prótesis glútea bilateral mediante cirugía en decúbito prono. Se identificó un desplazamiento del cojín posicionado en la cara y la cabecera no correspondía con la estructura ósea de la paciente. En sala de recuperación la paciente refirió pérdida visual en ojo izquierdo, por lo cual fue evaluada inmediatamente por Oftalmología encontrando una presión intraocular bilateral normal; un examen de fondo de ojo izquierdo evidenció múltiples áreas de desprendimiento de la retina. La tomografía con coherencia óptica confirmó la presencia de fluido subretiniano. Fue tratada con nepafenaco y prednisolona tópicas y evaluada a las 72 horas y a la semana, con resolución total del cuadro y agudeza visual corregida de $20 / 20$. No existen reportes en la literatura de desprendimiento seroso de retina posterior a cirugía en prono. Al evaluar el caso se asumió que la incorrecta posición del cojín y el tamaño inadecuado de la cabecera fueron factores que podrían haber contribuido a estasis venoso del flujo coroideo aumentando súbitamente la presión hidrostática en la coroides, lo que provocó el desprendimiento seroso de retina.

Palabras clave: Desprendimiento de retina; Posición prona; Procedimientos quirúrgicos operativos; Ceguera transitoria; Complicaciones.

\footnotetext{
Abstract

Prone position is used in surgery to access different anatomical structures of the patient. This position can be associated with postoperative visual loss in 0.05 to $1 \%$ of complications. Serous retinal detachment is the separation of the neurosensory retina from the retinal pigment epithelium by fluid accumulation without structural damage. This is the result of changes in the permeability and perfusion of the choroid. This is the case of a woman with an indication for removal of a bilateral gluteal prosthesis by surgery in the prone position. A displacement of the cushion positioned on
} 
2. Residente Cirugía general. Universidad CES, Medellín, Colombia.

3. Estudiante de Medicina, Universidad CES, Medellín, Colombia.
El desprendimiento seroso de retina es la separación de la retina neurosensorial y del epitelio pigmentario de la retina por acumulación de líquido, sin daño estructural, perdiéndose la adhesión dada por los procesos interdigitales apicales. the face was identified and the headboard did not correspond to the bone structure of the patient. In the recovery room, the patient reported visual loss in the left eye, which was immediately evaluated by Ophthalmology, finding a normal bilateral intraocular pressure. The left eye fundus examination revealed multiple areas of retinal detachment. Optical coherence tomography confirmed the presence of subretinal fluid. She was treated with topical nepafenac and prednisolone, and evaluated at 72 hours and a week, with total resolution of the condition and corrected visual acuity of 20/20. There are no reports in the literature of serous retinal detachment after prone surgery. When evaluating the case, it was assumed that the incorrect position of the cushion and the inadequate size of the bedside are factors that could have contributed to venous stasis of the choroidal flow, suddenly increasing the hydrostatic pressure in the choroid that caused the serous retinal detachment.

Keywords: Retinal detachment; Prone position; Minor surgical procedures; Transient blindness; Complications.

\section{Introducción}

La posición del paciente en decúbito prono es utilizada en cirugía para acceder a la fosa posterior del cráneo, cuello, columna vertebral, retroperitoneo, área perianal, y glúteos (1). Esta posición puede asociarse a pérdida visual postoperatoria en el 0,05 a $1 \%$ de las complicaciones reportadas (2). Entre estas, la neuropatía óptica isquémica es la más frecuente en el $89 \%$ de los casos con pérdida visual irreversible $(3,4)$. Otros diagnósticos diferenciales son oclusión de la arteria o la vena central de la retina, ceguera cortical y cierre agudo del ángulo esclerocorneal (5).

El desprendimiento seroso de retina es la separación de la retina neurosensorial y del epitelio pigmentario de la retina por acumulación de líquido, sin daño estructural $(6,7)$, perdiéndose la adhesión dada por los procesos interdigitales apicales (8). Algunas causas inflamatorias, vasculares y neoplásicas pueden generar cambios en la permeabilidad y en la perfusión de la coroides desencadenado el incremento del líquido intersticial en la coroides con extensión al espacio subretiniano $(6,9)$. Cuando el desprendimiento de retina compromete el polo posterior de la retina y el área macular, se asocia a disminución severa de la agudeza visual (10).

Se presenta el caso de una paciente con desprendimiento de retina con compromiso de la agudeza visual luego de cirugía en decúbito prono.

\section{Reporte del caso}

Una mujer de 40 años fue llevada a cirugía para el retiro de prótesis bilateral de biopolímeros en glúteos. La cirugía se llevó a cabo en posición de decúbito prono y bajo anestesia general. Entre sus antecedentes personales se destacaba mamoplastia de aumento tres meses antes, sin otros antecedentes de relevancia. En la consulta preanestésica se valoraron las pruebas, sin hallazgos patológicos.

En el examen físico no se encontraron alteraciones. Evaluada la vía aérea fue catalogada como grado I de Mallampati y clasificación ASA I según la American Society of Anesthesiologist. Luego de la inducción anestésica se posicionó a la paciente en decúbito prono con rodillas y caderas flejadas a $30^{\circ}$, ubicando un soporte almohadillado sobre las crestas iliacas, otro debajo del surco mamario y otro ubicado inmediatamente superior a las prótesis mamarias para evitar presión sobre las mismas. Se utilizó un soporte de espuma para la cabeza y se ubicó en posición neutra y lineal a 
Bajo dilatación ocular, el examen de fondo de ojo izquierdo evidenció múltiples áreas de desprendimientos serosos que comprometían el polo posterior y la periferia media. la columna vertebral. Este soporte era de un tamaño mayor para la estructura ósea de la paciente; sin embargo, no ocluía el área de la nariz y los ojos por lo que se decidió dejarse. Se evaluó de nuevo la posición del tubo sin encontrar desplazamiento.

Durante la cirugía se realizó monitoreo de presión arterial no invasiva, pulsoxímetro y capnografía; las variables hemodinámicas permanecieron estables presentando hipotensión controlada hasta finalizar el procedimiento, con una duración total de una hora y 28 minutos.

Al finalizar, se evidenciaron cambios en la posición de la paciente, tales como cabeza lateralizada que generaba presión sobre la órbita izquierda; igualmente, el soporte almohadillado utilizado en tórax, superior a las prótesis mamarias, estaba levemente desplazado hacia el cuello.

Se extubó en el quirófano y fue trasladada a sala de recuperación. En sala de recuperación la paciente refirió visión borrosa por el ojo izquierdo por lo que se evaluó fondo de ojo, sin encontrar hallazgos patológicos. Se aplicó lubricante ocular (carbómero) en forma de gel, sin ninguna mejoría, por lo que se decidió consultar a oftalmología.

La evaluación encontró agudeza visual en Logmar en ojo derecho fue de 0,0 (Snellen 20/20) y en ojo izquierdo de 0,2 (Snellen 20/32). Sin hallazgos a la evaluación con lámpara de hendidura y las pupilas eran reactivas. La presión intraocular era de $11 / 10 \mathrm{~mm} / \mathrm{Hg}$. Bajo dilatación ocular, el examen de fondo de ojo izquierdo evidenció múltiples áreas de desprendimientos serosos que comprometían el polo posterior y la periferia media. El área macular se encontraba respetada. El fondo de ojo derecho no mostraba alteraciones. Una tomografía de coherencia óptica identificó fluido subretinano (desprendimiento seroso de la neuroretina) en diferentes sectores del ojo izquierdo (figura 1).
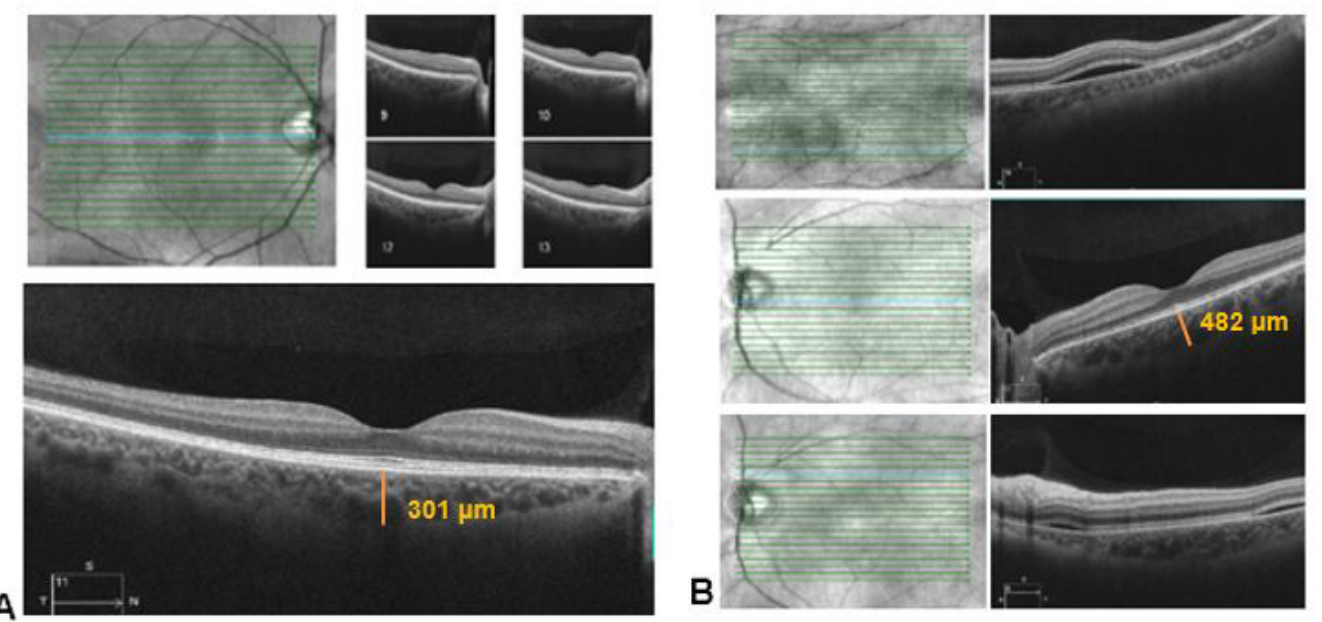

B

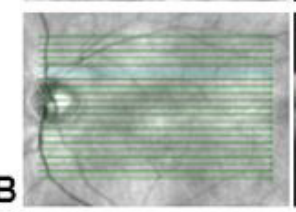

Figura 1. A). Tomografía de coherencia óptica de ojo derecho con grosor coroideo normal de 301 micras. B).Tomografía de coherencia óptica de ojo izquierdo. Se evidencia separación de las capas de la retina en relación con la coroides en diferentes ubicaciones de la retina; imagen de la fóvea evidencia ligero aumento del grosor coroideo de 482 micras en comparación con ojo derecho. 
Septiembre - diciembre de 2020 - Pág 218

Se indicó nepafenaco tópico al 0,1% una gota cada ocho horas y prednisolona tópica al $1 \%$ en dosis de una gota cada seis horas. Fue evaluada a las 72 horas y a la semana, observando reabsorción total del desprendimiento en todas las áreas comprometidas (figura 2). La agudeza visual final mejor corregida en ojo izquierdo fue 0,0 en la escala de LogMAR (Snellen 20/20).

Se identificó una inadecuada posición del soporte utilizado en el tórax, lo que pudo generar mayor presión intratorácica y disminución del gasto cardíaco, por la reducción en el retorno venoso, sumándose a las alteraciones hemodinámicas por posición en prono de manera independiente.
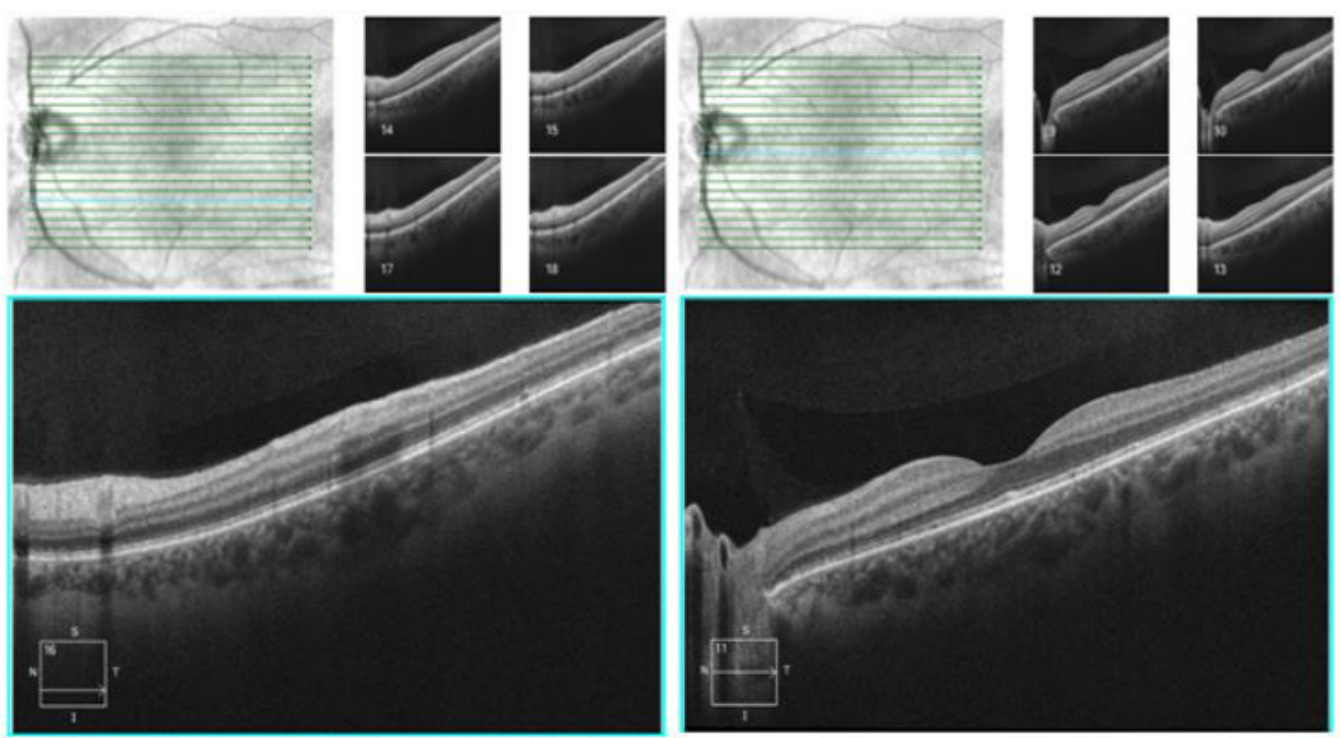

Figura 2. A). Tomografía de coherencia óptica de ojo derecho normal a las 72 horas. B). Tomografía de coherencia óptica de ojo izquierdo. Se evidencia completa resolución del desprendimiento de retina a las 72 horas luego del tratamiento.

\section{Discusión}

No conocemos otros reportes en la literatura de desprendimiento seroso de retina secundario a posición prono. En el análisis posterior del caso se identificó una inadecuada posición del soporte utilizado en el tórax, lo que pudo generar mayor presión intratorácica y disminución del gasto cardíaco, por la reducción en el retorno venoso, sumándose a las alteraciones hemodinámicas por posición en prono de manera independiente $(11,12)$. Se evidenció, además, que el soporte de la cabeza era de un tamaño mayor y no correspondía a la estructura ósea de la paciente, por lo que durante la cirugía la cabeza pudo haber rotado hacia la derecha y así comprimir externamente el ojo izquierdo, aumentando la estasis vascular (13).

Existen varios tipos de almohadillas disponibles diseñadas para la posición prono, la mayoría de gomaespuma, la cual sostiene la frente, las regiones malares y la barbilla, y tiene agujeros para los ojos, la nariz y la boca. En este caso la cara no siempre es visible, lo que hace difícil la vigilancia de los ojos; igualmente, los soportes en forma de herradura solo sostienen la frente y los pómulos; sin embargo, son rígidos y pueden ser peligrosos si la cabeza se mueve. Cualquiera que sea el método empleado para sujetar la cabeza, debe inspeccionarse frecuentemente la cara para asegurarse que el peso recae solo sobre las estructuras óseos y no sobre los ojos (13). Se considera que ambos factores generaron una estasis venosa del flujo coroideo, aumentando la presión hidrostática y su posterior redirección de flujo hacia el espacio subretiniano $(14,15)$. 
En pacientes llevados a cirugía en decúbito prono el posicionamiento debe ser más diligente por el compromiso de variables hemodinámicas dadas por la anestesia, utilizando apoyos adecuados para la contextura del paciente y la verificación de la posición durante todo el procedimiento.
A partir de un sistema de transporte activo el epitelio pigmentario de la retina moviliza fluido y solutos desde el espacio subretinal hacia la coroides, que al ser altamente vascular y permeable emplea fuerzas hidrostáticas y osmóticas para desplazar el líquido hasta el drenaje venoso (8). Un aumento de la presión hidrostática en la coroides genera un desbalance transitorio de este mecanismo (9).

Al reevaluar la tomografía de coherencia óptica se observó un incremento del grosor coroideo en el ojo izquierdo con respecto al derecho, lo cual podría explicar el incremento de fluido en la coroides en el ojo izquierdo. En este caso, el cuadro resolvió una vez se suspendió el estímulo causante.

Se adicionaron medidas de prevención luego del evento adverso, la adquisición de soportes faciales de gel que al ser moldeables permiten balancear el peso de la cabeza del paciente y evitar su movimiento.

\section{Conclusión}

En pacientes llevados a cirugía en decúbito prono el posicionamiento debe ser más diligente por el compromiso de variables hemodinámicas dadas por la anestesia, utilizando apoyos adecuados para la contextura del paciente y la verificación de la posición durante todo el procedimiento.

\section{Aspectos éticos}

Fue un reporte descriptivo retrospectivo, sin riesgo para la paciente. La paciente firmó el consentimiento informado. Los autores no declaran conflictos de interés.

\section{Bibliografía}

1. Breyer KEW. Patient Positioning and Associated Risks. In: Pardo MC MD, Miller RD MD, MS, editors. Basics of Anesthesia [Internet]. 2018. p. 321-36. Available from: https://www.clinicalkey.es/\#!/content/3-s2.0-B9780323401159000190

2. Kwee MM, Ho Y-H, Rozen WM. The prone position during surgery and its complications: a systematic review and evidence-based guidelines. Int Surg. 2015;100(2):292-303.

3. Gill B, Heavner JE. Postoperative visual loss associated with spine surgery. Eur Spine J. 2006;15(4):479-84.

4. Amorim Correa JL, Acioly MA. The Enigma of Orbital Compartment Syndrome After Lumbar Spine Surgery in the Prone Position: Case Report and Literature Review. World Neurosurgery. 2018;110:309-14.

5. Epstein NE. Perioperative visual loss following prone spinal surgery: A review. Surg Neurol Int. 2016;7(Suppl 13):S347-360.

6. Ghazi NG, Green WR. Pathology and pathogenesis of retinal detachment. Eye (Lond). 2002;16(4):411-21.

7. Steel D. Retinal detachment. BMJ Clin Evid [Internet]. 2014 Mar 3 [cited 2019 Jul 11];2014. Available from: https://www.ncbi.nlm.nih.gov/pmc/articles/ PMC3940167/ 
8. Thomas BJ, Albini TA. Serous Detachments of the neural retina. In: Yanoff M MD, Duker JS MD, editors. Ophthalmology [Internet]. 2019. p. 653-659.e1. Available from: https://www.clinicalkey.es/\#!/content/3-s2.0-B9780323528191003182

9. Wolfensberger TJ, Tufail A. Systemic disorders associated with detachment of the neurosensory retina and retinal pigment epithelium. Curr Opin Ophthalmol. 2000;11(6):455-61.

10. Corbett JJ, Chen J. The visual system. In: Fundamental neuroscience for basic and clinical applications [Internet]. Elsevier; 2018 [cited 2020 Aug 26]. p. 286-305.e1. Available from: https://linkinghub.elsevier.com/retrieve/pii/ B9780323396325000207

11. Edgcombe H, Carter K, Yarrow S. Anaesthesia in the prone position. British Journal of Anaesthesia. 2008;100(2):165-83.

12. Lin S, Hey HWD, Lau ETC, Tan K-A, Thambiah JS, Lau L-L, et al. Prevalence and predictors of pressure injuries from spine surgery in the prone position: do body morphological changes during deformity correction increase the risks? Spine. 2017;42(22):1730-6.

13. Cassorla L, Lee J-W. Colocación del paciente y riesgos relacionados. In: Miller RD MD, Cohen NH, Eriksson LI, Fleisher LA, Wiener-Kronish JP, Young WL, editors. Miller Anestesia [Internet]. 2016. p. 1240-65. Available from: https://www.clinicalkey.es/\#!/content/3-s2.0-B978849022927900041X

14. Pınar HU, Kaşdoğan ZEA, Bașaran B, Çöven İ, Karaca Ö, Doğan R. The effect of spinal versus general anesthesia on intraocular pressure in lumbar disc surgery in the prone position: A randomized, controlled clinical trial. Journal of Clinical Anesthesia. 2018;46:54-8.

15. Chui J, Craen RA. An update on the prone position: Continuing Professional Development. Can J Anesth/J Can Anesth. 2016;63(6):737-67. 